\title{
STUDIES IN THE PHYSIOLOGY OF BLOOD VESSELS IN MAN. APPARATUS AND METHODS. I. A SENSITIVE PLETHYS- MOSPHYGMOGRAPH FOR A PORTION OF THE FINGER ${ }^{1}$
}

\author{
BY ROY H. TURNER \\ (From the Department of Medicine, Tulane University of Louisiana, New Orleans)
}

(Received for publication May 4, 1937)

The apparatus I wish to describe was designed for the study of volume changes due to varying degrees of fullness of the smaller blood vessels in a portion of the human body, particularly for that portion of the finger distal to the major skin creases at the terminal finger joint.

The principal characteristics aimed at in devising the apparatus and method were: 1 . Accurate measurement should be possible for either quick volume changes due to the pulse when as small as $0.5 \mathrm{cu} . \mathrm{mm}$. and of slower changes in volume of relatively great magnitude, that is up to $800 \mathrm{cu}$. $\mathrm{mm}$. 2. Each type of measurement should be based on graphic records made simultaneously. 3 . In use, no influence of instrumental origin should modify the physiological phenomena under study, particularly by mechanical pressures causing constriction and consequent distension or emptying of blood vessels or by causing pain, fear, or other discomfort. The temperature, humidity and movement of the air around the skin should be normal. 4. The accuracy of measurements should not be impaired by change in room temperature. 5. The instrument should be dependable and easy to use. The apparatus about to be described seems to meet satisfactorily these requirements except those concerning skin environment and the effect of room temperature.

The apparatus, other than photokymograph and lamp, consists of a fine recorder (Figure 1), a coarse recorder and calibrator (Figure 4), a finger container cup and connecting tubes (Figure 5), all of which enclose a common space filled with air slightly above atmospheric pressure. The volume of the air space is susceptible to change by movements of the walls in only three separate portions of the apparatus, first by bulging or retraction of the walls (skin) of that portion of

1 Aided by grants from The David Trautman Schwartz Fund, The Josiah Macy, Jr. Foundation, and The Committee on Scientific Research of the American Medical Association. the air space which comes in contact with the body part being observed; second, by change in shape and size of the fine recording capsule; third, by lengthening or shortening the metal bellows in the coarse recorder. Whenever the air space is encroached upon by a slight swelling of the finger, the recorder capsule bulges by an almost equal volume. The volume increase of the capsule is slightly less than the volume increase of the finger due to the compression of the air in order to overcome the resistance of the capsule to distortion. If the swelling of the body part is of great magnitude, great distension of the capsule tends to occur, but is prevented by the intervention of the operator who, being warned by the wide excursion of the light beam, brings the system back to original volume and pressure by accommodating the air displaced from the finger cup within the coarse recorder where the volume of the bellows is increased until the fine recorder has returned to its zero range. A beam of light from one of the coarse recorder mirrors traces a record of this large volume change.

The three principal units of the apparatus which encloses the air space are connected by three lengths of rubber tubing leading to the three arms of a four-way metal connection from the fourth arm of which another length of rubber tubing makes connection with a stopcock which opens or closes the air space. Each of three lengths of rubber tubing is one foot long, and the fourth, that which leads to the finger container cup, is six feet long. The internal diameter of all tubing and connections is $1 / 16$ inch and the outside diameter $3 / 16$ inch. A thin coating of stopcock grease was always applied to the metal connection parts before inserting them into the rubber tubing. Leaks, which have given little trouble, are indicated by drift of the fine recorder beam. Constancy in the volume of the enclosed air space is dependent upon constancy in temperature. A 
change in temperature of $1^{\circ} \mathrm{C}$. produces a volume change of approximately 0.26 per cent, which for an air volume of $5 \mathrm{cc}$. would cause a volume change of $13 \mathrm{cu}$. $\mathrm{mm}$. The apparatus has been used solely in a room where the temperature was kept automatically at either $21.1^{\circ} \mathrm{C}$. or $23.9^{\circ} \mathrm{C}$. with a maximum variation of $\pm 0.3^{\circ} \mathrm{C}$. The apparatus functions to a certain extent as a radiometer, giving in response to hyperemia of the skin a beam deflection indicative of increased finger volume which is in part actually due to the increased temperature of the skin and the consequent increased temperature of the air within the finger container cup. However, the temperature effect is usually quite small as compared with the actual volume change of the finger.

The fine recorder. The fine recorder (Figure 1) is an optical capsule employing the well-known principles of the Frank segment capsule and differing chiefly in that stretching of the rubber membrane is largely avoided. Instead of stretching the rubber tambour over a rigid metal cup as is the usual procedure, a shallow flexible cup of thin rubber was used which permitted bulging of the membrane with less stretching. The capsule $(C$ and $C f$ ) was made of two circles ( $7 / 16$ inch in diameter) of sheet rubber (made by the anode method, Miller Rubber Company, Akron, Ohio) 0.007 inch thick stuck together at their peripheries. In the finished capsule, the front circle carried the mirror $(m)$ and the rear circle which had been slightly cupped before being joined to the front circle, served to exert slight stretch on the front membrane. In making the capsule, the two small pieces of sheet rubber were vulcanized to each other by a heated dull cork borer. The piece which was to serve as a cup was cemented to the squarely cut end of a piece of copper tubing $(t)$ (outside diameter $1 / 8$ inch) and the tube unsealed by inserting a hot wire into the lumen. The tube was slipped into a hole at the bottom of a shallow crater which had been turned in the surface of a small brass block. The piece of rubber was cupped by pressing the center to the desired depth in the crater. The front membrane was slightly stretched over the concave surface of the rear piece and the heated tube applied. At this stage all parts were held mechanically in proper alignment and the degree

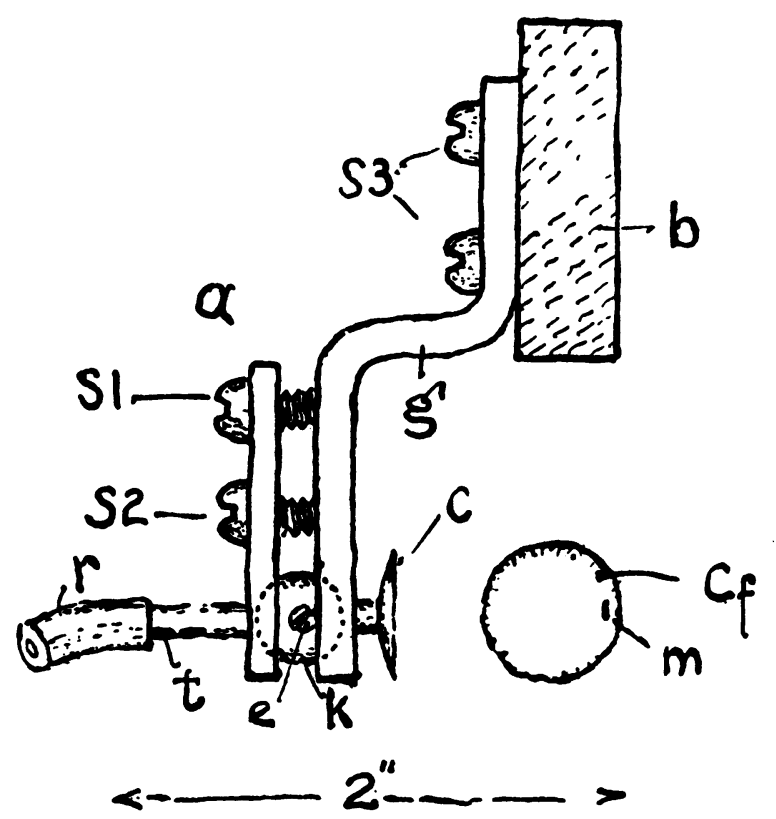

Fig. 1. Fine Recorder Capsule Unit

View from right side and in addition a front view of capsule $(C f)$ and mirror $(m)$. Rigid brass bar $(b)$ in cross section upon which different recording devices are mounted. Screws $(S 3)$ pass through vertical slots in part $(g)$ which allow adjustment vertically. $C=$ capsule side view. $t=$ copper tubing (outside diameter $=$ 1/8 inch). $K=$ ball through which tube passes; the ball fits into sockets in parts $(a)$ and $(g) . e=$ setscrew with countersunk head. $r=$ rubber tubing. $S 1=$ screw threaded into both $(a)$ and $(g)$ and serves as hinge for the two parts. $S 2=$ screw threaded only into $(g)$, adjustment of which tightens or loosens ball $(K)$ in socket permitting setting of filament image from mirror $(m)$ at proper natural zero, and also by rotation about axis of tube to adjustment of long axis of mirror to vertical.

of stretch of the front membrane controlled accurately by having it fastened to a metal ring through which, for the desired distance, could be thrust, by a screw mechanism, a smaller ring producing stretch of the desired degree. These parts as a unit were held in the lathe chuck, and the heated cork borer, its cutting edge accurately shaped, was carried by the tail piece of the lathe quickly and accurately through the opening of the inner ring. The two pieces of membrane were caught between the surface of the crater block and the edge of the heated tube and sealed to each other. Small leaks due to failure of vulcanization were sealed by passing them through a small drop of rubber cement two or three times with intervals 


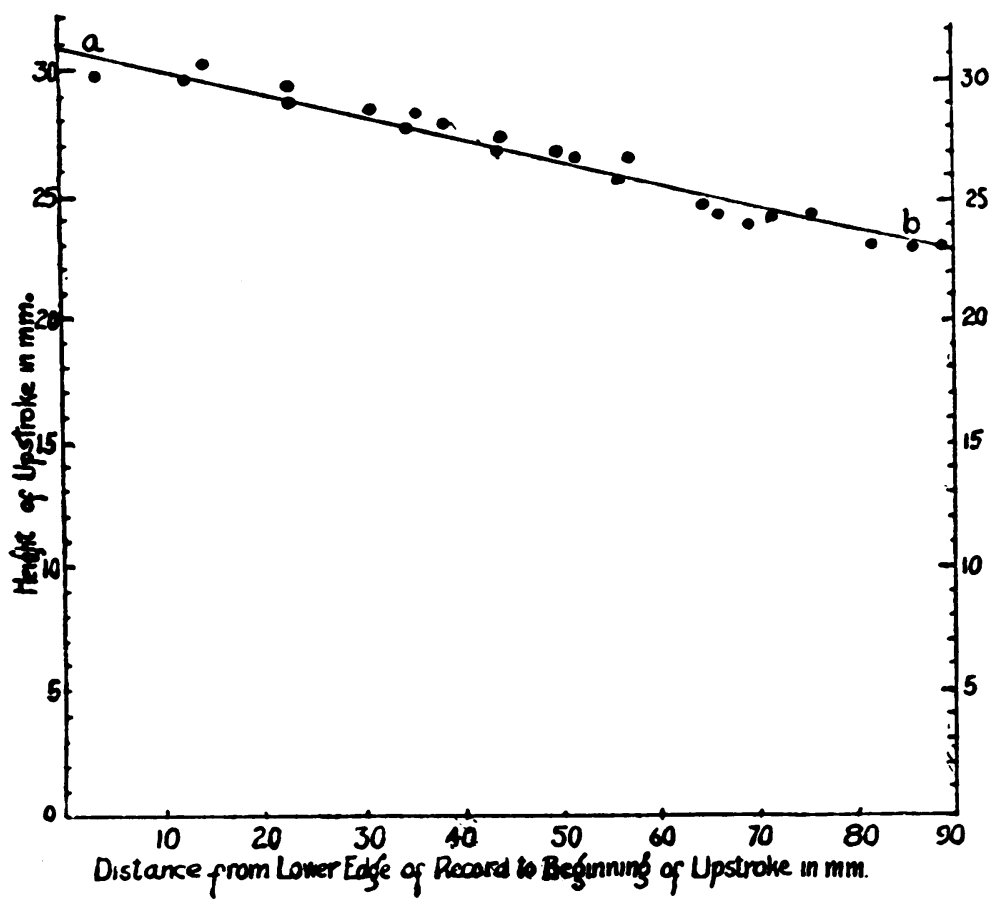

Fig. 2. Variation in Volume Response of the Fine Recorder Due to State of Fullness of the Capsule

Data for the graph were obtained by making 69 quick calibrations in groups of three distributed across the width of the photographic strip. Height of upstroke refers to beam deviations caused by diminution of calibrator volume by $5 \mathrm{cu}$. $\mathrm{mm}$., each dot representing the mean of 3 different deviations or upstrokes. The lower edge of the photographic record was to the right of the capsule during the process of recording. As the volume of the capsule increased the beam swung from right to left (upward on the finished record) and with increasing volume the size of the deviation per unit of volume change in the calibrator tended to diminish. Volume of the air space was approximately $7.0 \mathrm{cc}$. Owing to the fact that the natural zero point for the recording beam was at a point $40 \mathrm{~mm}$. from the right edge of the photographic strip while in the camera, a slight degree of inflation of the capsule had been accomplished before the beam fell on the strip.

between for drying. A rectangular mirror $(m)^{2}$ $3 / 64 \times 1 / 64$ inch was stuck with shellac to the front membrane about $1 / 18$ inch from the circumference with the long axis parallel to a chord of the circle. The capsule was mounted by a rigid support in front of the photokymograph, as shown in Figure 1. A straight filament lamp ${ }^{3}$ was mounted rigidly $4 \frac{1}{2}$ inches in front of and slightly below the mirror with filament vertical and side of bulb toward mirror and completely enclosed in a metal

\footnotetext{
2Westinghouse Electric \& Manufacturing Company No. 594186.

3 Westinghouse Electric \& Manufacturing Company No. 463604.
}

housing except for a narrow window which allowed the light to fall only upon the mirrors and the adjoining spaces. A photokymograph carrying bromide paper $120 \mathrm{~mm}$. wide and driven vertically downward was placed $211 / 2$ inches in front of the mirrors.

Individual capsules differed somewhat in sensitivity, ranging from 3 to $9 \mathrm{~mm}$. deflection for a volume change of $1 \mathrm{cu}$. $\mathrm{mm}$. Deflections are read to the nearest $0.1 \mathrm{~mm}$. on the record, which for a 5 to 1 calibration means reading to the nearest $0.05 \mathrm{cu} . \mathrm{mm}$. Capsules of somewhat different design have had a sensitivity of 14 to 1 . Such extreme sensitivity involves difficulties since small 


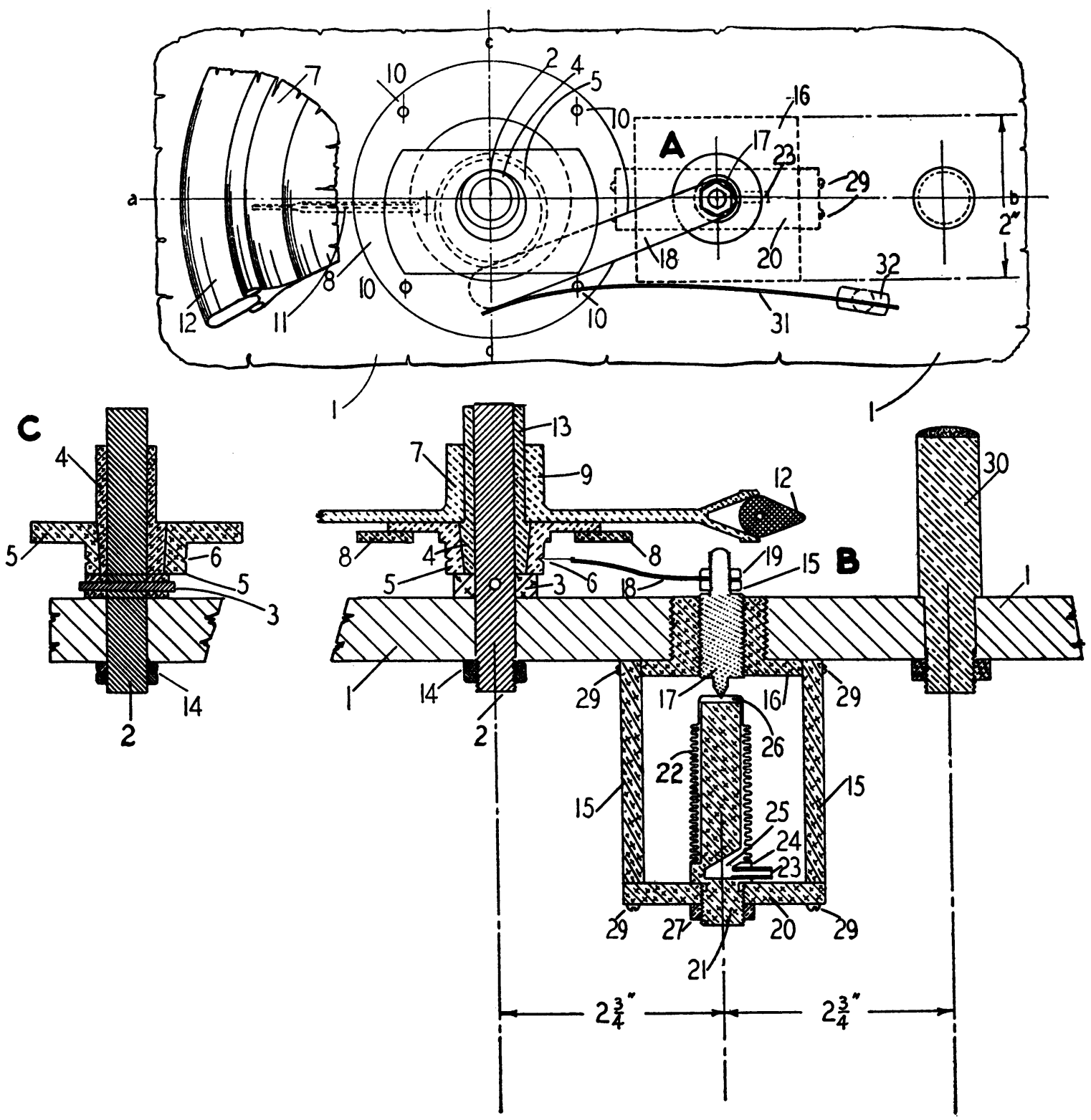

Fig. 3. Device for Producing Rhythmic Volume Changes of Desired Magnitude and at Varying FREQUENCIES

Volume changes are produced by shortening or lengthening of the metal bellows (22) by the screw (17) which is rotated back and forth through a variable angle maximum at $3^{\circ}$ by the lever (18) which rests in a circular v-shaped groove on the face of the cylinder (6) which functions as a variable eccentric which may be set so as to impart to lever (18) at point of contact a to-and-fro movement of from 0 to $1 / 4$ inch. For the tests mentioned in the accompanying article the apparatus was driven directly through a short piece of rubber tubing, one end of which was slipped over the motor shaft and a shaft-like projection bolted to the hub of the wheel (7).

Three partial views:

$A$. Top view with all except a fragment of drive wheel (7) and rubber tire (12) cut away.

$B$. Partial sectional view through $a-b$.

$C$. Sectional view of eccentric assembly through $c-d$. 
volume changes are likely to cause the beam to leave the photographic film.

The degree of proportionality between volume change and deflection of the recording bean throughout the recording range is shown in Figure 2. The deflections due to both the calibrator and pulse are routinely recorded so as to be centered upon the same longitudinal line on the photographic strip. Recording pulse volumes of greater or less amplitude than that of the calibration introduces a positive error on one side of the line and a negative error on the other which tend to neutralize each other. The tendency toward diminished deflection per unit volume change as the capsule volume increases is accentuated as the volume of the total air space of the apparatus increased, but is of little importance for volumes of less than $10 \mathrm{cc}$. Air pressures within the capsule, as we have used it, do not exceed $12 \mathrm{~mm}$. of water above atmospheric pressure.

- The maximum length of life of these capsules has not been determined. However, one has been in regular use for eleven months without evidence of deterioration.

So far as I am aware, no one has established such comprehensive criteria for the volume-frequency characteristics of a capsule designed for recording volume changes with minimal pressures in the system as exist for pressure recorders (1) in which volume changes are kept as low as pos-

All parts made of brass unless otherwise specified.

1. Steel base plate.

2. Steel shaft.

3. Cuff fastened to shaft with tapered steel pin.

4. Sleeve which acts as bearing for pulley; upper portion concentric with shaft and lower portion a tapered eccentric with center $1 / 10$ inch off that of shaft. This is the inner eccentric.

5. Outer eccentric with peripheral circle centered $1 / 16$ inch from that of inner eccentric. By rotation of outer eccentric upon inner eccentric the distance between the centers of the shaft (2) and the outer eccentric may be varied from 0 to $1 / 8$ inch. The circumference of the outer eccentric may impart a maximum motion of $1 / 4$ inch along the radius of a circle centered with shaft (2) to an object pressed properly against it.

6. Groove in outer eccentric in which rocker arm (18) rests.

7. Aluminum V-pulley fixed to (3) by 4 screws, one of which is (9).

8. Clamping ring. By tightening 4 screws (10) the outer eccentric is fixed to pulley (7) by friction, holding a given setting of outer eccentric indicated by pointer (11) on graduated scale on rim of pulley. The scale is graduated in cu. mm. volume change of bellows.

9. Setscrew.

10. Holes for screws.

11. Steel pointer for indicating on scale volume change in bellows per revolution of pulley.

12. Solid rubber tire which establishes driving contact with brass drum on motor shaft. (Motor and driving drum not shown.)

13. Cuff for holding moving parts on shaft.

14. Nuts.

15. Lateral pieces of bellows frame.

16. Top piece of bellows frame fixed by screwing into base (1) and receiving the screw (17).

17. Screw with threads 16 per inch, $1 / 4$ inch pitch, lead of 4 , rotated back and forth by rocker arm (18) through an arc up to $3^{\circ}$ as determined by the setting of outer eccentric (5).

18. Rocker arm of phosphor bronze $1 / 10$ inch thick.

19. Nuts.

20. Bottom piece of bellows frame through which bellows filler (21) is threaded and held by nuts (27).

21. Bellows filler for limiting dead space.

22. Metal bellows (Fulton-Sylphon, Number 107611-24B).

23. Piece of $1 / 8$ inch copper tubing soldered into hole (24) connecting with hole (25) establishing connection

27. Nuts. between the air space (26) within bellows (22) and the connecting tube.

29. Screws which hold together the 4 parts of bellows frame.

30. Upright shaft upon which is mounted driving motor.

31. Spring $1 / 32$ inch thick of phosphor bronze $1 / 2$ inch wide. Keeps rocker arm (18) pressed snugly into its groove on the outer eccentric.

32. Spring holder. The lower portion. 


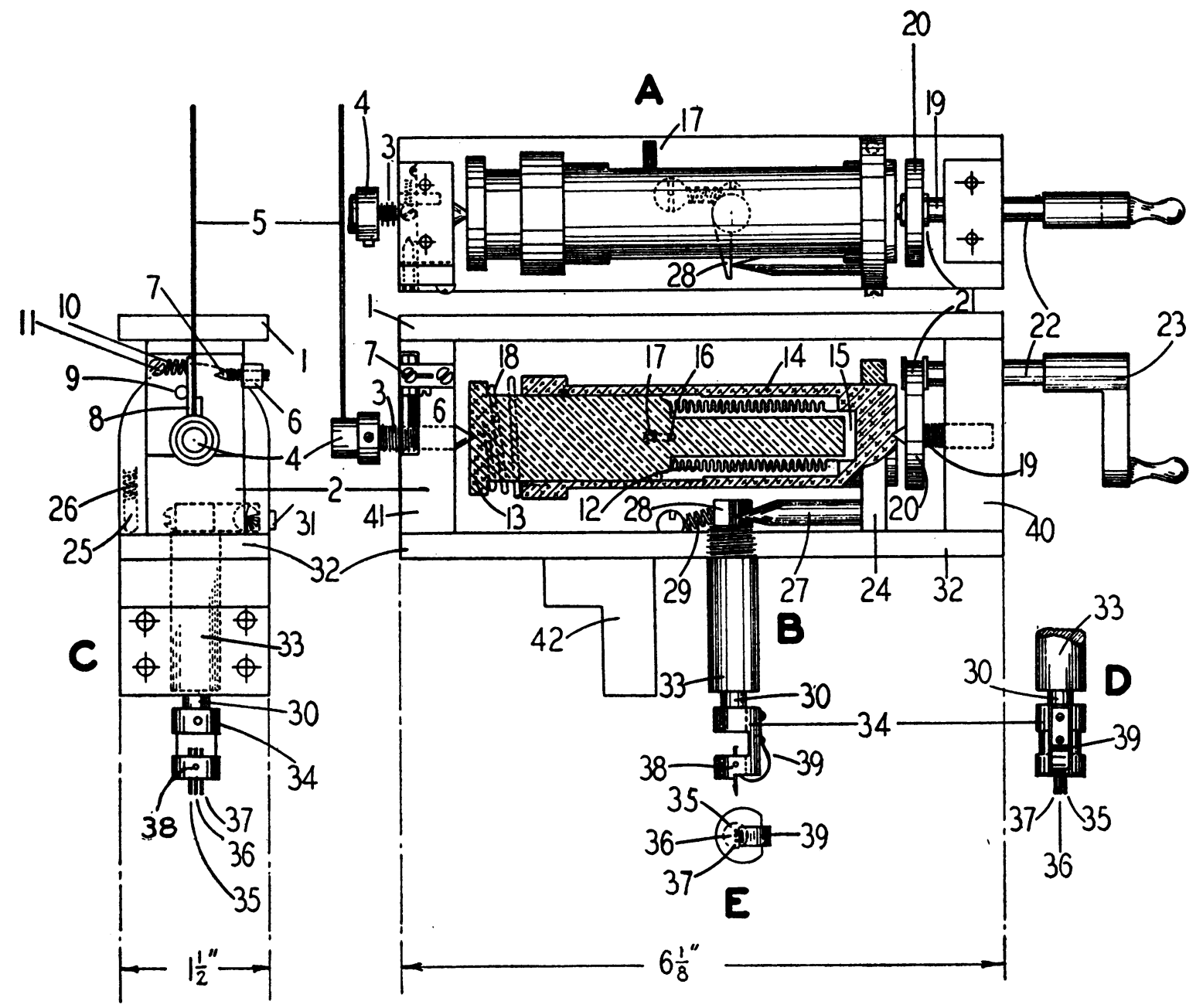

FRONT

Fig. 4. The Coarse Recorder and Quick Calibrator

$A$. View from above with top horizontal, (1) removed and quick calibrator level (5) and portion of cuff (4) cut away.

$B$. View from left side showing in section the bellows (12) and the male (13) and female (14) parts into which the two ends of the bellows are soldered.

$C$. View from the front.

$D$. View from rear showing only the mirror shaft assembly.

$E$. View of mirror assembly only, from below.

All parts made of brass unless otherwise specified.

1. Top piece of frame.

2. Front end piece of frame.

3. Screw, 20 threads per inch, for instantaneous calibrator.

4. Cuff.

5. Calibrator handle of number 18 music wire.

6. Split block for screw (7).

7. Adjustable stop of steel for instantaneous calibrator.

8. Steel lever passing through screw (3), movement limited by adjustable stop (7) and fixed stop (9).

10. Spring attached between steel lever (8) and screw (11).

12. Brass bellows (Fulton-Sylphon Number 107611-24B) length varied by the movement upon each other of two 
sible. It has seemed advisable to observe the response of the capsule to known equal changes in volume of the air space in another portion of the apparatus at widely varying frequencies. For the purpose of bringing about these volume changes at the desired frequencies it was necessary to design and build a special device. The construction and mode of operation are shown in Figure 3 and the accompanying legend. The capsule has been tested by joining the variable air space of this apparatus to the air spaces of the capsule and calibrator by means of the same rubber tubing used for connecting the finger cup. For each test equal rhythmic volume changes were employed at varying frequencies. In different tests the volume change varied from 2 to $10 \mathrm{cu}$. $\mathrm{mm}$. In a typical experiment using volume changes of constant size and at increasing frequencies, it was observed that at $\mathbf{5}$ cycles per second the amplitude recorded by the deflected light beam was $19.5 \mathrm{~mm}$. which increased with increasing frequencies so that at 10 cycles per second the amplitude was $23.5 \mathrm{~mm}$. where it maintained a high degree of constancy up to a frequency of 40 cycles per second when the amplitude of the deflections began to diminish and at 60 cycles per second had fallen to an amplitude of $12 \mathrm{~mm}$. The chief purpose for which this recorder was designed was the measurement of the volume changes due to the pulse in a portion of the finger. The most difficult task for such a device is the recording of the upstroke of the pulse wave which may occupy as little as 0.01 second, the equivalent of half of a 50 cycle wave. The behavior of the capsule was observed, using several technics, when it was required to make one quick change in volume. In one technic the capsule was connected to one of two short pieces of rubber tubing which were separated by a glass stopcock. The open end of the second piece of tubing was closed by a clamp and with the stopcock closed either positive or negative pressure was produced within the second piece of tubing by the use of another clamp. When the stopcock was quickly opened, there was a single quick deviation of the recorder beam without overshooting to a new position typically as follows. The entire deviation of the filament image was $37 \mathrm{~mm}$., 92 per cent (34 mm.) occurred in 0.016 second and 8 per cent $(3 \mathrm{~mm}$.) required 0.080 second. This is the behavior of a highly damped recorder. Using another technic, when a deviation of $55 \mathrm{~mm}$. oc-

parts (13 and 14) holding the two ends of bellows.

13. Male part and dead space filler soldered into open end of bellows.

14. Female part into which closed end of bellows was soldered.

15. Air space varied by length of bellows.

16. Hole leading from air space to rubber tubing slipped over tube (17).

18. Spring which tends to lengthen bellows, thrusting male part against screw (3) and female part against screw $(19,80$ threads per inch, left handed) which is rotated by gear $(20,32$ teeth) in mesh with gear (21, 6 teeth) on shaft (22) which is turned by handle (23).

24. Part soldered to (14) prevents rotation of bellows supports by sliding along bottom of frame (32) against which it is tightly pressed by pin (25) and spring (26).

27. Rod with eccentric point held in part (24) by screw (31); point presses against flat face of lever (28) which rotates shaft (30) which carries coarse recorder mirror. Lever 28 is held against point of rod (27) by spring (29).

33. Bearing for shaft (30). Cuff about 30 preventing upward movement of shaft not shown in drawing.

34. Mirror carrier block.

35, 36, 37. Mirror carrier rods of number 5 jewelers' pivot steel, lower ends cut at an angle of $7^{\circ}$ from axis of shaft (30) to receive mirrors.

38. Setscrew for fixing mirror rod (36).

39. Flat spring split to make 3 springs which prevent mirror carrier rods from falling out where screws are loosened.

40. Rear frame end piece.

41. Front frame end piece. Same as (2).

42. Bracket for fastening instrument to supporting horizontal bar by means of screws. The same bar also supports the fine recorder capsule. Both coarse and fine recorder mirrors (Westinghouse number 594186) receive light from same straight filament lamp (Westinghouse number 463604) from a distance of $41 / 2$ inches and both record on the same moving strip of bromide paper at a distance of $211 / 2$ inches without the use of lens except the cylindrical lens of the photokymograph. 
curred in less than 0.002 second there was some overshooting. In routine use the regular mode of quick calibration gives a volume change requiring 0.033 second which is the equivalent of one-half cycle at a frequency of 15 per second.

The coarse recorder. This unit consists of the coarse, or slow, recorder and the quick calibrator, the construction of which is shown in Figure 4 and the accompanying legend. The rods $(35,36$, 37) supporting the mirrors are so adjusted as to space evenly the three images of the lamp filament at such distances apart that, as the mirror shaft $(A, B, C 30)$ is rotated, one image does not leave the photographic record before the next arrives upon it. In this way the coarse recorder utilizes the equivalent of a photographic strip slightly less than three times the width of that actually employed. By rotating the rod with the eccentric point ( $A$ and $B 27$ ) the effective length of the lever $(A 28)$ can be varied so that the value in $\mathrm{cu} . \mathrm{mm}$. of volume of a given deviation of an image on the photographic record can be set at a convenient figure. For calibrating the coarse recorder the outlet was connected by rubber tubing to a pipette of $0.1 \mathrm{cc}$. capacity which could be read to 1 cu. mm., clamped in a horizontal position and containing a small drop of alcohol. By turning the crank ( $A$ and $B$ 23) volume change in the metal bellows $(B 12)$ was produced, displacing the drop along the lumen of the pipette. By changing the volume in units of $10 \mathrm{cu} . \mathrm{mm}$. and stopping the meniscus of the drop for a moment at each $10 \mathrm{cu}$. mm. mark on the pipette, a step-like line was produced on the record. The lateral displacement of the line at each step represented a record of a volume change of $10 \mathrm{cu} . \mathrm{mm}$. by that particular mirror and position on the photographic strip. By opening the system and putting the drop back to the starting end of the pipette such equal changes in volume were recorded with the image from each of the three mirrors for the entire width of photographic paper. No significant variation in calibration factor was found throughout the recording range. The setting routinely used gives $1 \mathrm{~mm}$. deflection of the filament image for a volume change of 0.18 cu. mm., and the records are measured to the nearest $0.1 \mathrm{~mm}$. The photokymograph used was made in our laboratory by Dr. W. H. Gillentine to whom I am indebted. The cylindrical lens is graduated in millimeters so that the developed record is ruled longitudinally in parallel lines 1 $\mathrm{mm}$. apart as is customary for electrocardiographic records. These lines are not photographed unless illumination is provided for that purpose. A small four candle power bulb is arranged in a housing alongside the capsule so as to provide sufficient light over the entire width of bromide paper to produce a shade of gray which contrasts with the white parallel lines and the black lines due to the filament images reflected from the mirrors.

The quick calibrator. Since the sensitivity of the fine recorder varies slightly with the volume of the enclosed air space in the apparatus, it is essential that its response to known volume change be determined each time the apparatus is newly applied to the study of a finger or whenever there is likelihood that volume change in the closed system of disturbing size has occurred. In most instances the enclosed air space has a volume of from 4 to $6 \mathrm{cc}$., and there is no striking change in calibration until the volume exceeds $10 \mathrm{cc}$. Calibration is accomplished by striking the spring handle ( $B$ and $C 5$ in Figure 4 ) of the quick calibrator with the finger so that the screw ( $A$ and $B 3)$ is rotated as far as stop $\left(\begin{array}{ll}C & 7) \\ \text { will }\end{array}\right.$ allow when the bending of the handle allows the finger to continue its swing releasing the mechanism and allowing it to return to its original state. In this way the bellows is quickly compressed giving a diminution in volume, then following a brief pause it is quickly returned to its original volume. The volume of the sensitive recorder capsule is suddenly increased then diminished by this procedure and a photographic record of the deviations of the filament image reflected from the capsule mirror provides the standard unit for determining what volume changes are represented by pulse complexes recorded by the same capsule. The calibration value is adjusted to a desired figure by the setting of the screw $(7 C)$ as determined by the movement of the alcohol drop when the calibration technic is executed slowly. We have used routinely a volume change of $5 \mathrm{cu}$. $\mathrm{mm}$.

The finger container cup. It is extremely diffcult to enclose a sharply defined portion of the human body in a rigid container so that pressure 


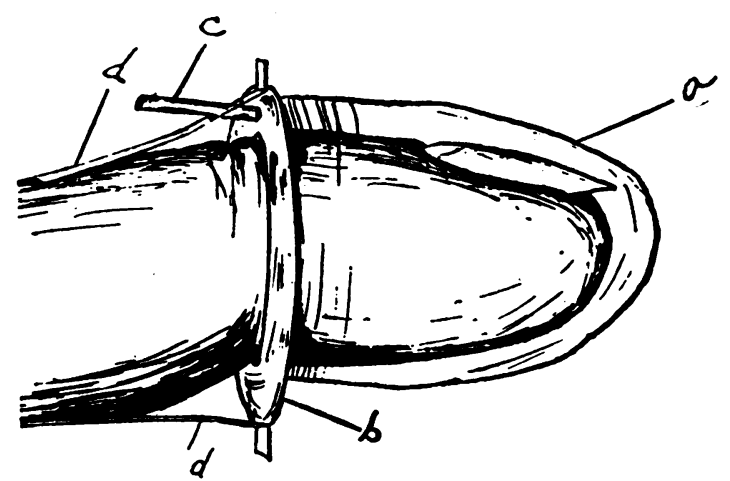

FIG. 5

a. Metal cup: In making the metal cup, $1 \times 6$ inches standard (tin-coated) collapsible metal barrels (Standard Specialty and Tube Company, New Brighton, $\mathrm{Pa}$.), such as used for toothpaste tubes, are cut into desired lengths and molded over carved wooden positive molds of sizes varying so that the internal surface of the cup shall be about $2 \mathrm{~mm}$. from the surfaces of the enclosed portion of the finger. After shaping the cup, the creases and cracks are sealed with cement (DuPont Household Cement).

b. Diaphragm: The diaphragm is cut into shape out of transparent Pyralin sheets (DuPont Viscoloid Co., Arlington, N. J.), 0.030 inch thick. A $1.5 \mathrm{~mm}$. opening is made for the continuation of the bore of the brass tube and about a $4 \mathrm{~mm}$. opening is made in the center. The latter opening is subsequently enlarged and properly shaped to fit the subject's finger.

c. Brass tube: The tube, approximately $2 \mathrm{~mm}$. external diameter, is made of sheet brass, 0.002 inch thick, with a soldered seam.

d. Cellulose acetate strips, approximately 0.25 inch wide and 0.008 inch thick for supporting the cup on the finger.

on, or tugging at the skin sufficient to disturb filling of small blood vessels does not occur. The terminal portion of the human finger has sharply defined landmarks and lends itself to a high degree of isolation and the blood supply is rich and varies widely under physiological and pathological influences. The cup which encloses the finger part should be light in weight and enclose a minimum air space which conforms exactly to the anatomical landmarks. The seal should be airtight and at the same time conform so easily to skin surfaces, even though the finger swell or shrink after the seal is applied, as to exert no disturbing pressure or tugging. The cup shown in Figure 5 seems to meet these requirements. I am indebted to Dr. G. E. Burch, Jr., who has made this unit a satisfactory part of the apparatus. The construction is explained in the legend for Figure 5. Several completely assembled units of different sizes, needing only shaping of the hole in the diaphragm to fit the finger, were kept available. When a subject presented himself for study the proper size cup was chosen and the opening in the diaphragm carefully shaped so that it fitted the finger loosely at the major dorsal and palmar creases at the terminal interphalangeal joint when the finger was comfortably flexed. The supports $(d)$ were adjusted so that they rested upon the finger without exerting any significant pressure or tugging and were stuck in place with rubber cement. The junction between the skin and the diaphragm was sealed air-tight with a non-constricting, elastic, adhesive composition which had been previously heated to body temperature. The composition consists of two parts of printers' roller compound and one part of a water-soluble, non-irritating, lubricating jelly. The rubber tubing leading to the recording apparatus is fixed to the dorsum of the subject's hand with adhesive tape, some slack being allowed between the cup and the adhesive tape. The complete finger unit weighs from 2 to 7 grams and contains about $3.5 \mathrm{cc}$. of air-space around the enclosed portion of the finger. Turning the finger gently so as to reverse the direction of the force of gravity causes no significant volume changes. Sudden movements of the fingers do cause quick transitory deflections of the fine recorder light beam. Unsatisfactory features of the finger container cup are that the air about the finger moves little, becomes heated, and soon develops high humidity, and unless the room is comfortably cool the sweat collecting in the air space may reach, in long experiments, an appreciable volume.

\section{SUM MARY}

An apparatus designed particularly for measuring volume changes due to the state of fullness of the blood vessels of a sharply defined portion of the human finger is described as to construction, mode of operation and working characteristics. The apparatus makes a graphic record of

$4 \mathrm{~K}-\mathrm{Y}$ Lubricating Jelly, Johnson and Johnson, New Brunswick, N. J. 
pulse volumes as small as $0.1 \mathrm{cu}$. $\mathrm{mm}$. and of gradual volume changes as great as $1000 \mathrm{cu} . \mathrm{mm}$. The pulse recorder which employs an optical capsule in which stretching of the rubber membrane is largely avoided shows high sensitivity, low moving mass, responds well to volume changes at a frequency up to 40 cycles per second and with diminished amplitude to 60 cycles per second and is well damped. The apparatus and method interfere with the body part under study to a minimal extent except through undesirable tempera- ture and humidity of the air in contact with the skin.

Acknowledgments. I am indebted to $\mathrm{Mr}$. George Johnson who constructed the apparatus and shared in all manipulations, and to my associates Drs. W. A. Sodeman and G. E. Burch, Jr., for valuable aid of many kinds.

\section{BIBLIOGRAPHY}

1. Wiggers, C. J., The Pressure Pulses in the Cardiovascular System. Longmans, Green and Co., London and New York, 1928. 